\title{
Congenital Posterior Urethral Diverticulum in a Male Child Case Report
}

\author{
Hasan Z1', Kumar Bindey², Kumar P³ \\ ${ }^{1}$ Dr. Zaheer Hasan, MBBS, MS, MCh. Assistant Professor, ${ }^{2}$ Dr. Bindey Kumar, MBBS, MS, MCh, Additional Professor, \\ ${ }^{3}$ Dr. Prem Kumar, MBBS, MD Radiology, Additional Professor. All from the department of Surgery, Indira Gandhi Institute \\ of Medical Sciences, Patna Bihar.
}

Address for correspondence: Dr. Zaheer Hasan, E-mail: drzaheerhasan@yahoo.com

\begin{abstract}
Bladder neck and posterior urethra are common sites for obstructive uropathy in children. Diverticula of posterior urethra are rare cause of obstruction in children. A six year old boy presented with features of bladder outflow obstruction since birth. Ultrasound findings were suggestive of posterior urethral valve. Micturating cystourethrogram and endoscopic examinations revealed posterior urethral diverticulum which was placed dorsally. Diverticulectomy and reconstruction of urethra was performed by midline perineal incision. This report emphasizes that a posterior urethral diverticulum may be considered in those cases where features are suggestive of posterior urethral valve bladder outflow obstruction.
\end{abstract}

Key words: Posterior urethral valve, Diverticulum, Diverticulectomy

\section{Introduction}

$D$ osterior urethral valve causing bladder outlet obstruction with back pressure changes is known in children but similar picture may be caused by congenital posterior urethral diverticula. We report a case of six year old boy who had features of bladder outflow obstruction. Micturating cystourethrogram revealed posterior urethral diverticulum which was suspected to have posterior urethral valve on ultrasonography, subsequently reconfirmed on cystourethroscopy.

\section{The Case}

A six year old male presented with complaints of straining while micturition, dribbling and sensation of incomplete emptying of urine with nocturnal enuresis for three years. There was no history of operative intervention. Physical examination was unremarkable. Laboratory evaluation revealed normal hemogram, blood urea $50 \mathrm{mg} / \mathrm{dl}$, serum creatinine $1.5 \mathrm{mg} / \mathrm{dl}$. Microscopic examination of urine revealed pus cells and growth in urine culture. Ultrasonography revealed thickened, trabeculated bladder and dilated posterior urethra. The patient was stabilized with urethral catheter and antibiotic cover. Micturating cystourethrogram revealed cystic dilatation in the region of posterior urethra which was placed dorsally resulting in bladder outflow obstruction with large postvoid residual urine volume (Figure 1). Cystourethroscopy revealed a diverticulum in the region of posterior urethra distal to verumontanum. Intra venous urography showed both kidneys normal without any dilatation of upper tract. Diverticulum was approached through midline perineal incision. Cystoscope was advanced to locate extent of lesion thereby facilitating dissection. Dorsally placed diverticulum was rotated ventrally by hooking it with infant feeding tube. Diverticulectomy and urethroplasty was done over the infant feeding tube with the help of vicryl 5-0 suture along with reinforcement of corpous spongiosum (Figure 2). Post operatively patient was voiding with good urinary stream with insignifant post void residual urine. In one year follow up in outpatient department the patient was asymptomatic.

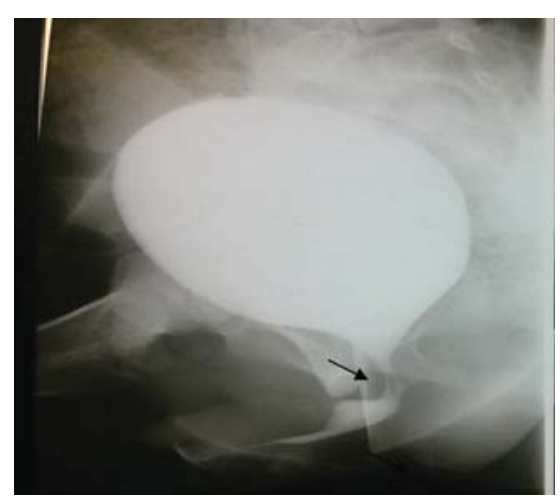

Fig 1: Photograph, MCU showing posterior urethral diverticulum. 


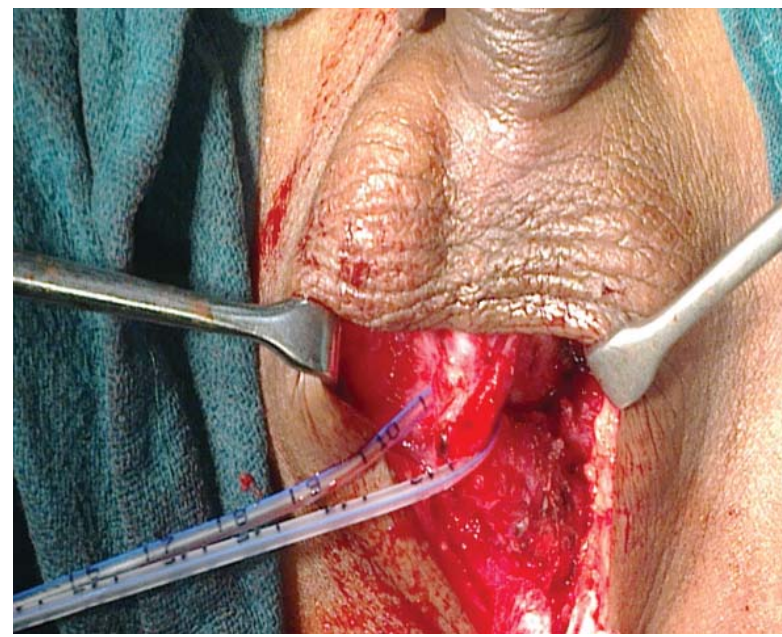

Fig 2: Photographs showing posterior urethral wall diverticulum hooked by Infant feeding tube

\section{Discussion}

Urethral diverticulum is defined as saccular envaginations of urethral mucosa. In contrary to anterior urethral diverticulum, posterior urethral diverticulum is generally acquired in origin. In a review of 95 diverticulae of posterior urethra, only six were of congenital origin ${ }^{1}$. Trauma of different types in an elderly multiparous female give rise to acquired posterior urethral diverticulum ${ }^{2}$. Etiology for congenital posterior urethral diverticulum is proposed that it is due to faulty or incomplete fusion of a segment of urethral plate. Congenital etiology is supported on the basis of back pressure changes in the urinary bladder without any predisposing factor. Proposed etiopathogenesis of posterior urethral diverticulum is that it can swell up significantly during micturition compressing bladder neck and urethra from posterior aspect resulting in outlet obstruction and its antecedent complications ${ }^{3}$.

We have noticed that posterior urethral diverticulum was deficient of corpous spongiosum. Common differential diagnosis of posterior urethral diverticulum are mullerian duct cyst and utricular cyst. The differentiating point of posterior urethral diverticulum with mullerian duct cyst and utricular cyst are opening which was paramedian and occupies distal to verumontanum in contrast to midline position in the later cysts. Congenital posterior urethral diverticulum may present at birth and in grown up children. Saurabh Agrawal et.al has reported posterior utethral diverticulum even in adult patient ${ }^{4}$. Similar case was reported by Shahram Mousavi et,al in a nineteen year old male patient ${ }^{5}$. Presenting features of posterior urethral diverticulum vary from asymptomatic patient to different features of lower urinary tract symptoms. Some neglected cases may present with calculi, persistent infection and even adenocarcinoma. In most cases of congenital posterior urethral diverticulum, diverticulum is placed ventrally ${ }^{6}$ but in the present case it was placed dorsally which is unique.

\section{Conclusion}

A high index of suspicion is required in order to diagnose posterior urethral diverticulum especially in bladder outlet obstruction cases. It is always should be kept in differential diagnosis where the diagnosis of posterior urethral valve is made.

\section{References}

1. Wachsberg RH, Sebastiano LL, Sullivan BC, Irwin R. Posterior urethral diverticulum presenting as a midline prostatic cyst: Sonographic and MRI appearance. Abdomen Imaging 1995;20:70-1.

2. PateVA, Bunts RC. Urethral diverticula in paraplegics. J Urol 1951;65:108 -23.

3. Nghiem HT, Kellman GM, Sandberg SA, Craig BM. Cystic lesions of the prostate. Radiographics 1990;10:635-5.

4. Saurabh Agrawal, M. S. A. Ansari, R. Kapoor, and D. Dubey. Congenital posterior urethral diverticula causing bladder outlet obstruction in a young male. Indian J Urol 2008;24(3):414-415.

5. Shahram Mousavi, Abdolrasoul Mehrsai, Mohammadreza Nikoobakht, Amir Reza Abedi, Sepehr Salem, Gholamreza Pourmand. A Giant Congenital Posterior Urethral Diverticulum Associated with Renal Dysplasia. Urol J 2006;4:247-9.

6. Gerald H.Jordan,Steven M Schlossberg in surgery of the penis and urethra :In Walsh,Retik,Vaughan,Wein Campbells'Urology. Eighth's edition 2002 pp3902.

\section{How to cite this article?}

Hasan Z, Kumar Bindey, Kumar P. Congenital Posterior Urethral Diverticulum in a Male Child Case Report. J Nepal Paediatr Soc 2012;32(3):257-258. 